\title{
GLAD!
}

Revue sur le langage, le genre, les sexualités

11 | 2021

Archives, genre, sexualités, discours

\section{Manon Garcia (dir). 2021. Philosophie féministe. Patriarcat, savoirs, justice}

\section{Vanina Mozziconacci}

\section{(2) OpenEdition}

9 Journals

Édition électronique

URL : https://journals.openedition.org/glad/3395

DOI : $10.4000 /$ glad.3395

ISSN : 2551-0819

Éditeur

Association GSL

Référence électronique

Vanina Mozziconacci, « Manon Garcia (dir). 2021. Philosophie féministe. Patriarcat, savoirs, justice», GLAD! [En ligne], 11 | 2021, mis en ligne le 20 décembre 2021, consulté le 29 janvier 2022. URL : http:// journals.openedition.org/glad/3395 ; DOI : https://doi.org/10.4000/glad.3395

Ce document a été généré automatiquement le 29 janvier 2022.

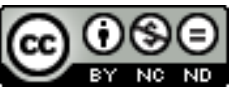

La revue GLAD! est mise à disposition selon les termes de la Licence Creative Commons Attribution Pas d'Utilisation Commerciale - Pas de Modification 4.0 International. 


\title{
Manon Garcia (dir). 2021. Philosophie féministe. Patriarcat, savoirs, justice
}

\author{
Vanina Mozziconacci
}

\section{RÉFÉRENCE}

Manon Garcia (dir). 2021. Philosophie féministe. Patriarcat, savoirs, justice, Paris : Vrin. 458 pages.

Publié dans la collection «Textes clés » de Vrin, qui présente des domaines classiques tels que «Philosophie du langage » ou « Esthétique » mais qui contient également des volumes plus inattendus comme Philosophie du végétal ou Éthique des affaires, l'ouvrage dirigé par Manon Garcia, en étant porté par cette maison d'édition de référence dans le champ $^{1}$, permet à la philosophie féministe de gagner en reconnaissance française (et il serait temps ! comme la chercheuse le souligne en introduction). Le principe de la collection est simple: un.e spécialiste choisit une dizaine de courts textes jugés représentatifs d'un domaine, qui sont classés en quatre ou cinq thématiques, et pour lesquels elle ou il écrit une introduction générale sur le sujet et des introductions spécifiques par sous-partie. L'ouvrage en question est ainsi organisé autour de quatre grands chapitres : "Femmes, féminisme et philosophie " (avec un texte de Michèle Le Dœuff et un autre de Nancy Bauer), "Épistémologies féministes » (contenant trois textes, écrits par Sandra Harding, Sally Haslanger et Geneviève Fraisse), « Féminisme et philosophie politique » (qui renvoie à Mary Wollstonecraft, Marilyn Frye et Christine Delphy) et enfin "Féminisme, universalisme et intersectionnalité » (pour les textes de Susan Moller Okin et Uma Narayan).

Elle-même chercheuse en philosophie féministe, Garcia publie cet ouvrage après la parution de son On ne nait pas soumise, on le devient, un essai qui, par son accueil très positif, scientifique comme grand public ${ }^{2}$, a fortement contribué à (re)mettre la philosophie féministe en bonne place au sein des intérêts politique et scientifique en France. Plus tard, en octobre 2021, elle publie La conversation des sexes. Philosophie du 
consentement, toujours chez Flammarion - l'essai a donc bien été transformé. Toutefois, le fait que la chercheuse travaille aux États-Unis (elle est enseignante-chercheuse à Harvard puis à Yale) et non depuis un poste dans l'Université française - pour l'instant - montre que ce travail de légitimation n'a rien d'évident dans l'espace académique hexagonal.

Garcia est claire sur les intentions et partis-pris qui ont guidé sa sélection : rappelant qu'il est impossible sur un si petit échantillon d'embrasser la richesse du champ, elle précise «avoir fait le choix de ne pas reproduire de[s] textes facilement accessibles $^{3}$ » (p. 26), c'est pourquoi les écrits de Simone de Beauvoir, de Monique Wittig, d'Audre Lorde ou encore de bell hooks ne se trouvent pas dans le volume. Cela explique aussi la part belle laissée aux traductions inédites dans l'ouvrage (mais uniquement depuis l'anglais ${ }^{4}$ ). Les textes introductifs sont particulièrement clairs et didactiques; ils résument avec concision mais sans simplification des enjeux aussi complexes que le rapport entre intersectionnalité et identity politics (p.379), la fausse neutralité de l'individu en termes de genre au sein de certaines théories de philosophie politique (p.22) ou encore les différentes branches de l'épistémologie féministe (p.126). Ces introductions sont également l'occasion pour Garcia d'indiquer des références particulièrement bienvenues en note, certaines classiques et d'autres plus méconnues, pour prolonger la réflexion. Le volume mérite donc d'être lu non comme un panorama exhaustif - ni même forcément représentatif - mais plutôt comme l'occasion de saisir en quoi la rencontre entre féminisme et philosophie déjoue des représentations communes de l'une comme de l'autre. En effet, la plupart des textes choisis sont remarquables par leur puissance critique, par leur singularité d'écriture et par la concrétude de leur engagement.

Du fait du contexte de publication de cette recension, je choisis de prendre pour fil directeur de mon compte-rendu la question du langage, question qu'on retrouve dans plusieurs textes sous des formes différentes, mais qui semble à chaque fois rappeler un principe important: les philosophes féministes ont une conscience vigilante que ces choses qu'elles manipulent sont des mots avant d'être des concepts ou des idées. Il me semble intéressant de mettre en avant tous les jeux avec/sur/de langage qu'une telle approche rend possible. Autre décision: $\mathrm{j}$ 'entre dans les textes par des thématiques plutôt que par le plan de l'ouvrage.

\section{Langage ordinaire}

$5 \quad$ Nancy Bauer résume la tension qui traverse le concept d'une philosophie féministe par le fait qu'« il faut opérer à la fois au niveau des concepts ordinaires - le niveau, après tout, auquel le féminisme se situe - et au niveau de la philosophie - où ces concepts ordinaires sont remis en question » (p.112-113). Et en effet, rien d'étonnant à ce que l'ordinaire se manifeste par le langage dans la philosophie féministe, puisqu'elle se doit de s'intéresser à un pan important de ce qui a été considéré - et continue d'être considéré - comme ordinaire donc vulgaire, ordinaire donc banal et sans intérêt philosophique : les petites choses de la vie quotidienne et routinière que sont le care, le travail domestique ou encore le corps des femmes.

Cela se manifeste notamment par un ton d'écriture: Christine Delphy mêle une exigence de démonstration soutenue avec un style relâché fait de petites phrases comme «Je sentais bien que quelque chose n'allait pas» (p.347), "Cette notion 
[patriarcat], il faut la remplir, et cela ne se fait que peu à peu» (p. 359), «j'ajoute les mots "ici et maintenant" qui font toute la différence» (p.360) - qui donnent l'impression d'être en conversation avec elle. Quoi de plus logique que de faire signe vers l'ordinaire pour un texte qui explique pourquoi elle en est venue à « regarder du côté de la famille et du travail qui y est fait » (p.350) jusqu'à conceptualiser le «mode de production domestique ». Pour reprendre une formulation de Sandra Laugier, la pensée féministe est par excellence celle qui «appelle notre attention sur ce qui est juste sous nos yeux, mais que nous ne voyons pas, par manque d'attention tout simplement, ou mépris », dans la " réalité ordinaire ${ }^{5}$ ». De là une langue qui va avec, et qui refuse le style continument jargonnant traditionnellement si prisé en philosophie.

Objectivité «forte » [strong objectivity], écrit Sandra Harding. L'adjectif forte est un mot simple, pour un concept finalement assez compliqué, qui revient à revendiquer à la fois le caractère situé des savoirs tout en rejetant subjectivisme, relativisme, perspectivisme et pluralisme (p. 159). Mais justement : dans l'expression objectivité forte, le terme fort vise à insister sur le fait qu'on évalue toujours les savoirs à l'aune de l'objectivité, tout en statuant que : «Le problème avec la conception traditionnelle de l'objectivité n'est pas qu'elle est trop "rigoureuse" ou trop "objectivante" comme certain.e.s l'ont avancé, mais qu'elle n'est pas assez rigoureuse ou objectivante : elle est trop faible pour accomplir ne serait-ce que les objectifs pour lesquels elle a été conçue » (p. 134). Cette force est à comprendre comme une exigence redoublée, celle du « défi que constitue le fait d'apprendre à penser depuis la perspective de plus d'une vie lorsque ces vies sont en conflit avec les autres» (p. 170). Cette exigence peut sembler " écrasante » (la force devient une pression qui broie certaines prétentions) lorsqu'on se contente d'une vision traditionnelle de l'objectivité : par exemple, celle qui consiste à croire pouvoir mettre sa propre situation entre parenthèses pour dégager une pensée universelle, comme un tour de passe-passe ou "truc divin»[God Trick], selon l'expression de Donna Haraway, qui formule elle aussi un gros problème dans un petit mot.

\section{Jeux de mots}

8 Si le titre du texte de Nancy Bauer mobilise un nom de figure de style («La philosophie féministe est-elle un oxymore?») et s'écarte ainsi d'un usage ordinaire de la langue pour faire signe vers une certaine technicité, on ne peut que remarquer le jeu auquel elle se prête en détournant des références érudites comme prétexte d'une énumération (" La philosophie première, Le Deuxième sexe et la troisième vague »).

9 Ainsi les autrices féministes ne se privent pas de jeux de mots, pour l'humour (omniprésent dans le texte de Michèle Le Dœuff) ou le plaisir ludique. Chez Geneviève Fraisse, c'est la paronomase avec l'épreuve/la preuve qui lui permet de lier histoire, philosophie et carrière politique puisque selon elle, parcourir l'Histoire est « une mise à l'épreuve conceptuelle» (p. 287) : on lit dans le livre du monde mais on y fait aussi ses preuves.

Il y a aussi des jeux de mots sérieux, puisque tout le texte de Sally Haslanger vise à montrer les liens réels entre deux termes aux connotations opposées et dont on pourrait croire qu'ils concernent des domaines radicalement différents : objectivité et objectification. L'idéal épistémologique auquel le premier terme renvoie semble en effet n'avoir rien de commun - hormis sa racine - avec le phénomène aliénant et réifiant 
vers lequel le second fait signe. Sans adhérer à une certaine misologie défendue dans le texte, on peut reconnaitre quelques liens entre ces deux réalités mis au jour par le regard féministe de Haslanger.

\section{Métaphores filées}

11 Il est remarquable que la figure de style de la métaphore filée se retrouve dans plusieurs textes choisis du volume, avec à chaque fois un rôle central. Il est significatif que le texte de Le Dœuff qui ouvre le volume soit tiré d'un ouvrage intitulé L'imaginaire philosophique, ouvrage qui cherche notamment à montrer que la philosophie n'est pas seulement peuplée de concepts mais également d'images, qui ne sont ni des vestiges d'une pensée "pré-philosophique » ni des concessions faites au nom de la pédagogie, mais bien des éléments opératoires de la démonstration philosophique elle-même. Ces images sont parfois des images de «LA» femme - qui permettent par exemple à un Rousseau ou à un Sartre de colmater les brèches de leur démonstration avec le plâtre de la misogynie ${ }^{6}$ - images auxquelles les femmes sont confrontées mais dans lesquelles elles n'ont pas à se reconnaitre, rappelle Le Dœuff. Contre un « toupet métaphysique (et logocentrique) » très masculin (p. 74) et sa raison molaire, elle suggère une pratique de la philosophie qui ne s'arc-boute pas contre sa « frange de non-savoir » (p. 75).

La métaphore de la cage à oiseau est par exemple essentielle chez Marilyn Frye. Dans son texte, elle cherche à définir avec précision le terme " oppression ", terme qui « est progressivement vidé de son sens » et "utilisé comme s'il pouvait s'étendre à toute expérience humaine de limitation ou de souffrance» (p. 323). Un tel usage n'est pas seulement relaché ou vague mais peut avoir des conséquences politiques dangereuses : en oubliant le critère de la dimension systémique de l'oppression et en s'autorisant à l'utiliser pour qualifier toute expérience individuelle d'hostilité ou de mal-être, il devient possible de construire des fictions comme "le racisme anti-blanc" (ou reverse racism), le " sexisme anti-homme », etc ${ }^{7}$. D'où la nécessité d'introduire l'idée de système - cette idée, abstraite, est rendue concrète par l'image de la cage à oiseau finement développée par Frye: "Imaginez une cage à oiseau. Si vous regardez de très près seulement un des barreaux de la cage, vous ne pouvez pas voir les autres barreaux. (...) même si jour après jour, vous inspectez chacun des barreaux un à un, il est possible que vous ne voyiez toujours pas ce qui empêcherait un oiseau de les franchir. (...) c'est seulement quand vous reculez, (...) que vous adoptez une vision macroscopique de la cage tout entière, que vous pouvez voir pourquoi l'oiseau ne va nulle part» (p. 328). Le caractère opératoire de la métaphore est confirmé lorsque l'autrice la remobilise pour expliquer en quoi la galanterie (et plus particulièrement le fait de tenir la porte aux femmes) relève du sexisme, car pour saisir la signification du rituel, il ne faut pas se concentrer "sur l'événement en lui-même, dans toute sa singularité ", avec une "perspective myope » (p. 331) mais voir comment il est lié à un système d'oppression plus large.

13 De même, dans le texte de Uma Narayan, la métaphore des "spectres", celle des «souliers" (la première partie du texte s'intitule «Spectres de l'autre femme ou comment ne pas se mettre dans les souliers d'une autre ») ainsi que celle du "bagage » jouent des rôles clefs pour critiquer un certain "impérialisme de l'imagination" (p. 413). Je reviens sur les deux premières. L'impérialisme de l'imagination consiste à se représenter une femme «Autre» (en l'occurrence «une femme du Tiers Monde ») 
depuis une perspective ethnocentrée et dominante, soit en s'identifiant complètement à elle, soit au contraire en projetant une différence totalisante. Ces alternatives prennent la forme de deux «spectres » dans le texte : le spectre de la prisonnière du patriarcat (qui se voit imposer des formes d'oppression contre sa volonté et contre son consentement) et le spectre de la dupe du patriarcat (qui s'auto-impose ces formes d'oppression, qui souscrit entièrement aux normes patriarcales de sa culture). D'après Narayan, ces deux spectres naissent de deux élans éthiques. Le premier est le résultat de «l'injonction courante incitant à s'imaginer dans les souliers de l'Autre - mais conduit à identifier les pieds de l'Autre femme aux nôtres, comme s'ils étaient susceptibles d'être pincés ou liés exactement aux mêmes endroits que les nôtres; l'on échoue par là à imaginer quel effet cela ferait d'être l'Autre femme, avec ses pieds à elle à l'intérieur de ses souliers» (p. 412-413). Le second élan éthique «implique bien que l'on s'efforce de prendre en compte le fait que l'Autre femme est façonnée par des circonstances, des engagements et un contexte différents du nôtre (...) qu'il est possible que ses souliers lui aillent différemment qu'à nous » (p. 413). Mais cette intuition pèche par le fait qu'elle "se figure les pieds de l'Autre femme comme s'ils se vouaient entièrement à la forme de ses souliers - comme des pieds qui, ou bien n'éprouveraient pas le moindre frottement au contact de ceux-ci, ou bien seraient déterminés à les porter au prix d'innombrables cloques " (Ibid.). La concrétude de la métaphore poussée jusqu'au bout de certains détails apparemment «triviaux» et douloureusement évocateurs ne va pas sans rappeler les " concepts à la sueur " (sweaty concepts) de Sara Ahmed, à savoir des concepts qui portent en eux la « description d'un corps qui n'est pas chez lui dans le monde ${ }^{8} "$.

Des images donc, mais qui ne sont pas des fantasmes, pour reprendre ce qu'écrit par ailleurs Le Dœuff : "Ce n'est pas la peine de me raconter les fantasmes, cela ne m'intéresse pas! Ils ont fait ça pendant vingt-cinq siècles, les confrères. Leur stock est bien suffisant pour l'analyse. Donc si vous parlez des femmes, parlez des femmes qui passent dans la rue, de ma vieille voisine de palier d'autrefois ${ }^{9}$. »

\section{Récits}

L'allégorie de la colporteuse (qui se compose avec mouvement, horizontalité, balluchon, etc.) permet à Fraisse de mettre en forme son parcours féministe et de souligner l'importance de l'historicité; elle n'est pas la seule à vouloir raconter le chemin tracé; plusieurs chercheuses féministes françaises soulignent ainsi l'importance des récits de/dans la recherche ${ }^{10}$.

16 De façon analogue, au lieu de la grande Histoire de LA philosophie, on peut suivre une (petite) histoire d'une philosophie. Je ne crois pas trahir la pensée de Nancy Bauer en la résumant ainsi, lorsqu'elle affirme que simone de Beauvoir est un exemple paradigmatique de prise en charge d'un paradoxe fondamental qui traverse toute philosophie féministe, tiraillée entre le quotidien et la métaphysique (p. 113). Reprendre les concepts « en réponse à sa propre expérience " revient, chez Beauvoir, à faire ce que Bauer appelle "un récit de femme» (p.118). Un récit dans lequel on ne cesse de retisser la pensée à ce qui lui a donné naissance, pour «l'empêcher de se détacher de ses propres intérêts » (p.119). On retrouve d'une certaine façon ici l'analyse que faisait par ailleurs Le Dœuff de la philosophie beauvoirienne, qui, contrairement à l'existentialisme sartrien, ne vise pas à boucler un système, mais où «la valeur 
commande le descriptif ${ }^{11}$ " car c'est à partir d'une situation concrète et située - et reconnue comme telle - qu'on philosophe. Le féminisme est alors le lieu d'un modèle pour la philosophie qui en fait un «mode de transformation et d'expression de soi » (p. 119) dans lequel conceptualiser et raconter vont de pair.

\section{NOTES}

1. À noter toutefois que si Vrin bénéficie d'un certain prestige, le travail éditorial pourrait être amélioré : plusieurs coquilles émaillent le texte, et ce défaut n'est pas propre à ce volume...

2. Voir les nombreuses recensions ici : http://www.manon-garcia.com/press

3. Elle ajoute toutefois «à l'exception de celui de Christine Delphy, indispensable pour l'analyse du travail domestique ". En effet, ce dernier texte mais aussi celui de Geneviève Fraisse (dont on retrouve des propos reprenant les mêmes idées dans un ouvrage récent intitulé Philosophie et féminisme) restent des travaux assez connus et diffusés. À noter que le texte de Michèle Le Dœuff est depuis peu disponible en ebook grâce au projet Gallica de numérisation des indisponibles : https://gallica.bnf.fr/ark:/12148/bpt6k33468943.texteImage\#

4. Traductions qui ont été réalisées pour la plupart par de jeunes chercheur.e.s en philosophie spécialistes du féminisme ou du genre.

5. LAUGIER, Sandra. 2009. «L'éthique comme politique de l'ordinaire » Multitudes 37-38 (2-3) : 80-88.

6. Pour une analyse de la façon dont la conceptualisation de la mauvaise foi sartrienne est pétrie de sexisme, voir LE DCEUFF, Michèle. 2008. L'Étude et le rouet: des femmes, de la philosophie, etc. Paris : Éditions du Seuil.

7. Sur la lecture individualisante de l'oppression et ses écueils, voir MOHANTY, Chandra Talpade. 1989. «On Race and Voice: Challenges for Liberal Education in the 1990s " Cultural Critique $14: 179-208$.

8. AHMED, Sara. 2014. «Sweaty concepts » [En ligne], consulté le 7 décembre 2021. URL : https:// feministkilljoys.com/2014/02/22/sweaty-concepts/

9. LE DEUFF, Michèle. 2020. « Michèle Le Dœuff, une philosophe féministe. Cheveux courts, idées longues » Nouvelles Questions Féministes 39(1) : 98-115.

10. Dans la continuité des faiseuses d'histoire, Mélody Faury raconte sa recherche sur son carnet Hypothèses L'infusoir (https://infusoir.hypotheses.org/category/autoethnographie-de-la-these) et Marys Hertiman, quant à elle, rappelle la nécessité de « rapporter ou raconter brièvement le contexte de recherche et de rédaction dans les publications scientifiques » et de "dévoiler dans l'introduction de la thèse la manière dont nous composons la recherche avec la vie personnelle, les discussions formelles et informelles avec des pair.e.s et des personnes éloignées de notre étude.... En indiquant aussi les difficultés dans le parcours de recherche »: https:// lesjaseuses.hypotheses.org/2584

11. L'Étude et le rouet, Id. p. 101. 
INDEX

Thèmes : Actualités 